\title{
Figure-Ground Organization in Visual Cortex for Natural Scenes
}

\author{
ㄴ) Jonathan R. Williford, ${ }^{1}$ and ${ }^{1}$ Rüdiger von der Heydt ${ }^{2}$
}

DOI:http://dx.doi.org/10.1523/ENEURO.0127-16.2016

${ }^{1}$ Netherlands Institute for Neuroscience, 1105 BA Amsterdam, Netherlands, and ${ }^{2}$ Johns Hopkins University, Baltimore, MD, 21218

\begin{abstract}
Figure-ground organization and border-ownership assignment are essential for understanding natural scenes. It has been shown that many neurons in the macaque visual cortex signal border-ownership in displays of simple geometric shapes such as squares, but how well these neurons resolve border-ownership in natural scenes is not known. We studied area V2 neurons in behaving macaques with static images of complex natural scenes. We found that about half of the neurons were border-ownership selective for contours in natural scenes, and this selectivity originated from the image context. The border-ownership signals emerged within 70 ms after stimulus onset, only $\sim 30 \mathrm{~ms}$ after response onset. A substantial fraction of neurons were highly consistent across scenes. Thus, the cortical mechanisms of figure-ground organization are fast and efficient even in images of complex natural scenes. Understanding how the brain performs this task so fast remains a challenge.
\end{abstract}

Key words: figure-ground; macaque; natural scenes; single units; visual cortex; visual perception

\section{Significance Statement}

Here we show, for the first time, that neurons in primate visual area V2 signal border-ownership for objects in complex natural scenes. Surprisingly, these signals appear as early as the border-ownership signals for simple figure displays. In fact, they emerge well before object selective activity appears in the inferior temporal cortex, which rules out feedback from that region as an explanation. Thus, "objectness" is detected by extremely fast mechanisms that do not depend on feedback from the known object-recognition centers.

\section{Introduction}

Many visual tasks depend fundamentally on our visual system being able to organize low-level image features together as objects. This is a challenging problem for images of natural scenes, in which object contours are buried in luminance and color variations produced by surface structure, illumination, and shadows, and features from different objects are cluttered because of interposition in space. How the visual system is able to accomplish this is a major unresolved question of visual neuroscience.
Received May 13, 2016; accepted December 2, 2016; First published December 15, 2016.

Authors report no conflict of interest.

Author Contributions: JRW: designed research, performed research, analyzed data, wrote the paper. RVDH: designed research, analyzed data, wrote the paper.
Border-ownership coding, discovered by Zhou et al. (2000), is an early neural correlate of this perceptual organization (Williford and von der Heydt, 2013). Zhou et al. (2000) found that when an edge of a figure, such as a square, is aligned to the classical receptive field (CRF) of neurons in macaque visual cortex, some neurons will fire at a higher rate when the figure appears on one side of the CRF compared with the opposing side, even when the stimulus is locally ambiguous about the side of the figure. The underlying figure definition mechanisms may provide

Office of Naval Research, National Institutes of Health.

Acknowledgments: We thank Hee-kyoung Ko for help with annotation of the natural scenes and helpful discussions.

Correspondence should be addressed to Jonathan R. Williford, Netherlands Institute for Neuroscience, Meibergdreef 47, 1105 BA Amsterdam, Netherlands. E-mail: jonathan.r.williford@gmail.com. 
the structure for object-based attention (Qiu et al., 2007). However, border-ownership coding has not yet been demonstrated for natural scenes. The same is true for other neural correlates of figure-ground organization such as the enhancement of activity over figure regions (Lamme, 1995; Zipser et al., 1996; Lee et al., 1998; Marcus and Van Essen, 2002).

The goal of the present study was to close this gap of knowledge. We did not know what result to expect. On the one hand, images of natural scenes are rich in information that might be used for figure-ground definition. On the other hand, these images are infinitely more complex than displays of simple geometrical figures. Models of border-ownership coding were mostly designed for geometrical figures (Zhaoping, 2005; Sakai and Nishimura, 2006; Craft et al., 2007), and when such models were tested on complex natural scenes, their performance was modest (Sakai et al., 2012; Russell et al., 2014).

Primate visual systems have no difficulty in segregating figure and ground in complex scenes, and it is commonly assumed that this is based on object knowledge. Thus, it is often assumed that border-ownership signals for complex natural scenes would depend on prior shape processing. Because shape-selective neurons in the inferior temporal (IT) cortex start to respond only $\sim 80 \mathrm{~ms}$ after stimulus onset and become shape selective even later (Brincat and Connor, 2006), this hypothesis predicts that border-ownership signals for natural scenes should be delayed compared with those for simple geometrical figures, which appear at $\sim 60-80 \mathrm{~ms}$ (Sugihara et al., 2011).

We report here that a subset of neurons in visual area V2 are selective for border-ownership in images of natural scenes. When the CRF of such a neuron aligns with an occluding contour, the neuron will fire at a higher rate on average when the occluding object is located on the neuron's preferred border-ownership side. Some neurons signal border-ownership consistently across different objects and scenes. The border-ownership signals are mainly driven by image context, whereas an image patch covering the CRF produces only a small transient signal. Surprisingly, border-ownership signals for natural scenes emerge as early as $70 \mathrm{~ms}$ after stimulus onset, which contradicts the traditional view which attributes image understanding to shape processing at levels much higher in the hierarchy.

\section{Materials and Methods}

We studied neurons in the visual cortices of three male rhesus macaques (Macaca mulatta). All procedures conformed to National Institutes of Health and U.S. Department of Agriculture guidelines as verified by the Animal Care and Use Committee of Johns Hopkins University.

DOI:http://dx.doi.org/10.1523/ENEURO.0127-16.2016

Copyright @ 2016 Williford and von der Heydt

This is an open-access article distributed under the terms of the Creative Commons Attribution 4.0 International, which permits unrestricted use, distribution and reproduction in any medium provided that the original work is properly attributed.

\section{Preparation}

Three small head posts for head fixation were implanted in the skull, and a recording chamber was placed over the visual cortex of each hemisphere under general anesthesia.

\section{Recording procedures}

Isolated neuronal activity was recorded extracellularly with glass-coated platinum-iridium microelectrodes (Pt-lr 0.1 - $\mathrm{mm}$ diameter, etched taper $\sim 0.1$, impedance 3-9 $\mathrm{M} \Omega$ at $1 \mathrm{kHz}$ ) that were inserted through the dura mater. A spike time detection system (Alpha Omega MSD 3.22) was used.

Most of the V2 neurons were located in the lunate sulcus after passing through $\mathrm{V} 1$; the remaining neurons were located in the lip of the postlunate gyrus. The eccentricities of the receptive fields ranged from 0.53 to 4.9 degrees of visual angle (median of 2.5).

\section{Stimuli and experimental design \\ Stimulus display}

The stimuli were presented to the monkeys with either a 21-inch EIZO FlexScan T965 or a ViewSonic G220fb color monitor. Both had the refresh rate set to $100 \mathrm{~Hz}$ and resolution of $1600 \times 1200$. The monitors were viewed at a distance of $1 \mathrm{~m}$ and subtended $21 \times 16$ degrees of the visual field. The luminous outputs of the monitors were linearized. The images of the natural scenes were gamma corrected with the equation $V_{\text {out }}=V_{\text {in }}^{2.2}$ for the linearized displays.

\section{Recording of gaze direction}

The direction of gaze was recorded for one eye by corneal reflection or pupil tracking using an infrared video system (Iscan ETL-200) that was aligned with the axis of the eye via an infrared-reflecting mirror. The system recorded direction of gaze with a resolution of $0.08 \times 0.16$ degrees of visual angle, although the accuracy was lowered by noise and drifts in the signal.

\section{Behavioral design}

All data were collected using a fixation paradigm. Monkeys were given a juice reward for keeping the eye position signal within 1 visual degree of the center of a fixation point for 3-4 s.

\section{Mapping procedures}

After isolating a cell, its CRF was manually mapped with luminance bars, luminance edges, drifting gratings, and/or rectangles, depending on the CRF properties of the neuron. Any region of the visual field where a stimulus elicited a clear response was considered part of the CRF. The radius of the CRF was used to define the patch condition of natural scene stimuli, described below. Color and orientation were varied to determine the optimal stimulus. The manual mapping was typically confirmed by a position test that systematically moved a luminance stepedge or bar in randomized order.

The orientation preference was first tested in steps of $30^{\circ}\left(0-330^{\circ}\right)$. Often the orientation was fine-tuned using smaller steps over a more focused range. Only cells that were orientation selective were included in the results. 
This was determined by using the orientation modulation index when orientation tests were presented or by manual mapping when the orientation preference was obvious. The orientation modulation index was calculated by

$$
\mathrm{OMI}=\frac{\left(R_{\theta}-R_{\theta \perp}\right)}{\left(R_{\theta}+R_{\theta \perp}\right)},
$$

where $R_{\theta}$ and $R_{\theta \perp}$ are mean responses to the preferred and orthogonal orientations. Only cells that had an orientation modulation index of at least 0.20 were included in the analysis.

\section{Standard square border-ownership test}

The border-ownership signal was first assessed using the standard square border-ownership test as described previously (Qiu and von der Heydt, 2005). This test presents a square with one of the edges centered over the CRF of the cell and the square rotated such that the orientation of the border matches the cell's preferred orientation. The square and the background are defined by two colors: gray $\left(28 \mathrm{~cd} / \mathrm{m}^{2}\right)$ and the preferred color of the cell (or a color that elicited a strong response). The assignment of each of these colors to the square and the background were counterbalanced to separate out the effect of local contrast from the border-ownership coding. Two sizes of squares were used: $3^{\circ}$ and $8^{\circ}$. A uniform background with the mean of these two colors was displayed between stimulus presentations.

For the standard square border-ownership test and the natural scene border-ownership test (described below), multiple stimuli were presented within a behavioral fixation period. Each stimulus was presented for $300 \mathrm{~ms}$, and a uniform screen was shown for $200 \mathrm{~ms}$ between the stimulus presentations, whose color was set to the mean stimulus color within the CRF.

\section{Natural scene border-ownership test}

We used images from the Berkeley Segmentation Dataset 300 (Martin et al., 2001) and one additional image (Paolo Neo, http://www.public-domain-image.com/freeimages/flora-plants/fruits/apple-pictures/red-apple-fromtop). Before data collection, we labeled many points in the images that lay on the occluding contours of objects, recording position, orientation of contour, and side of the occluding object. Each neuron was tested with a number of images aligning one of these points in the center of the CRF. Custom software was used to assist in accurately positioning the points. We call each test point in an image a "scene." Not all of the recorded neurons were presented with the same scenes. Fig. 1 shows 51 points denoted in the 32 images that were used most frequently in the experiments. The exact subset varied between monkeys, because some scenes seemed to distract a given monkey more than others from the fixation task, leading to frequent abortion of trials. Typically, only one point per image was tested, although sometimes there were more. In early experiments, we selected, for each neuron, points where the contour orientation was within $5^{\circ}$ of the neuron's preferred orientation and rotated the images to match the preferred orientation exactly. In later experiments, we selected a subset of scenes (shown in Fig. 1) and applied arbitrary rotation. This enabled us to test the same scenes across multiple cells.

In the initial experiments, when we selected points based on how closely their contours matched the recorded neuron's preferred orientation, we included more complex images. However, when we later concentrated on a smaller subset of points (shown in Fig. 1), we avoided points with high curvature, contour junctions, and thin objects, where both sides of the objects could fall within the receptive field.

For each scene, we manipulated border-ownership, edge contrast polarity, and image context (Fig. 2).

\section{Border-ownership}

To manipulate the side of the object owning the border in a given scene, the image was rotated $180^{\circ}$ about the center of the CRF. For example, in Fig. 2, the fish is to the upper right of the border, which is Cell 1's preferred side-of-object before rotation. After rotation, the fish is on the opposite side.

\section{Edge contrast polarity}

Rotation of the images by $180^{\circ}$ changes the local contrast polarity within the CRF, since the local contrast rotates with the image. To control for this, we performed a transformation of the RGB color space that inverted the mean colors within the CRF on either side of the border. The color inversion transformation we used for a given scene (scene point), $s$, is

$$
T\left(x_{i}, s\right)=\left(\overline{x_{1}^{s}}+\overline{x_{2}^{s}}\right)-x_{i},
$$

where $\overline{X_{1}^{s}}$ and $\overline{X_{2}^{s}}$ are the average colors on each side of the border within the approximate receptive field radius, $r$; and $x_{i}$ is the color of the pixel that is being transformed. The regions $x_{1}^{s}$ and $x_{2}^{s}$ were segmented by hand. This is similar to the common color inversion transformation; however, the color space is inverted around the mean colors within the CRF instead of the midpoint of the color space.

\section{Patch}

To separate the effect of the local stimulus within the CRF (which is driving the response) from the modulation by the context, we showed both full images and local patches that covered the CRF. The stimulus within the approximate receptive field radius, $r$, was exactly the same for the full images and the patches. Outside this radius, the stimulus faded into the background using the complementary error function:

$$
\alpha=\left\{\begin{array}{ll}
1 & d<r \\
\operatorname{erfc}[k(d-r)], & d<r
\end{array},\right.
$$

where $d$ is the distance from the center of the receptive field in pixels, and $k$ was set to 1.8 .

Each of these factors (border-ownership, edgecontrast-polarity, patch) is binary, such that there are a total of eight variations of each scene. All of the stimuli were presented in pseudorandom order.

The background color surrounding the full images and patches, and the display color during the interstimulus 


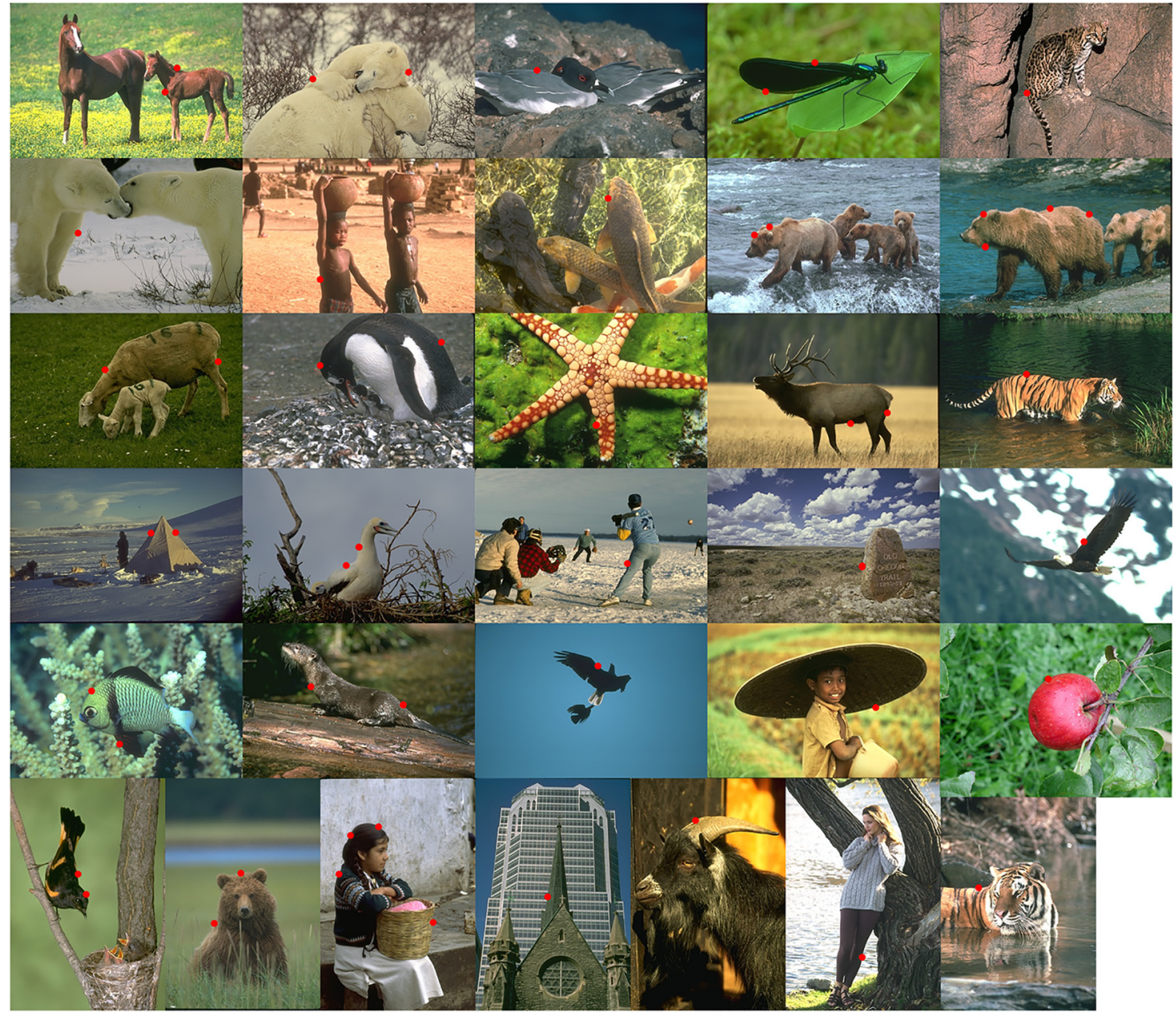

Figure 1. Example scenes used for testing border-ownership selectivity of neurons. Selected points on occluding contours (marked here by red dots for illustration) were centered in the receptive field of the neuron to be studied. The examples show the most frequently used scenes, with the number of neurons tested per scene ranging from 90 (top left) to 10 (bottom right). All the images are from the Berkeley Segmentation Dataset (Martin et al., 2001), except for the image of the apple on the lower right (Paolo Neo, http://www.public-domain-image.com/free-images/flora-plants/fruits/apple-pictures/red-apple-from-top) .

intervals for a given scene, was the average color within the area of the CRF:

$$
B G(s)=\frac{\left(\overline{x_{1}^{s}}+\overline{x_{2}^{s}}\right)}{2}
$$

This definition of background color for each scene is analogous to the definition of the interstimulus color used in the standard square test.

\section{Data analysis}

\section{Analysis of spike counts}

We used linear models to analyze each cell separately with R software (R Development Core Team, 2008; RRID: SCR_001905). The spike counts between 40 and $300 \mathrm{~ms}$ of stimulus onset were transformed by the Anscombe transform, $f(x)=\sqrt{x+3 / 8}$.

This transform approximately converts Poisson data to Gaussian distributions. Type II repeated-measures ANOVA ( $R$ car package; Fox and Weisberg, 2011) was used to measure the effect of border-ownership and its significance. A three-way fixed-effects model was used for the standard square test data (border-ownership $\times$ edge-contrastpolarity $\times$ size), and a four-way model for the natural scenes data (border-ownership $\times$ edge-contrast-polarity $\times$ patch $\times$ scene ID). Here, the variable "patch" specifies whether the full image was presented or only the patch over the CRF, as explained above. All the factors were binomial except scene ID, which had multiple levels and could also vary between cells. Both analyses were based on factorial design and included all interactions. The context influence on the border-ownership signal in natural image was defined as the interaction between borderownership and patch.

To determine consistency of border-ownership signals and compare their relative strengths across different stimulus conditions, we plotted the border-ownership effects of the full images, the patches, and the context alone, as a function of the border-ownership effects of the standard square test, and in each case fitted a line through the origin using orthogonal least-squares regression. Orthogonal regression was used to treat both variables symmet- 

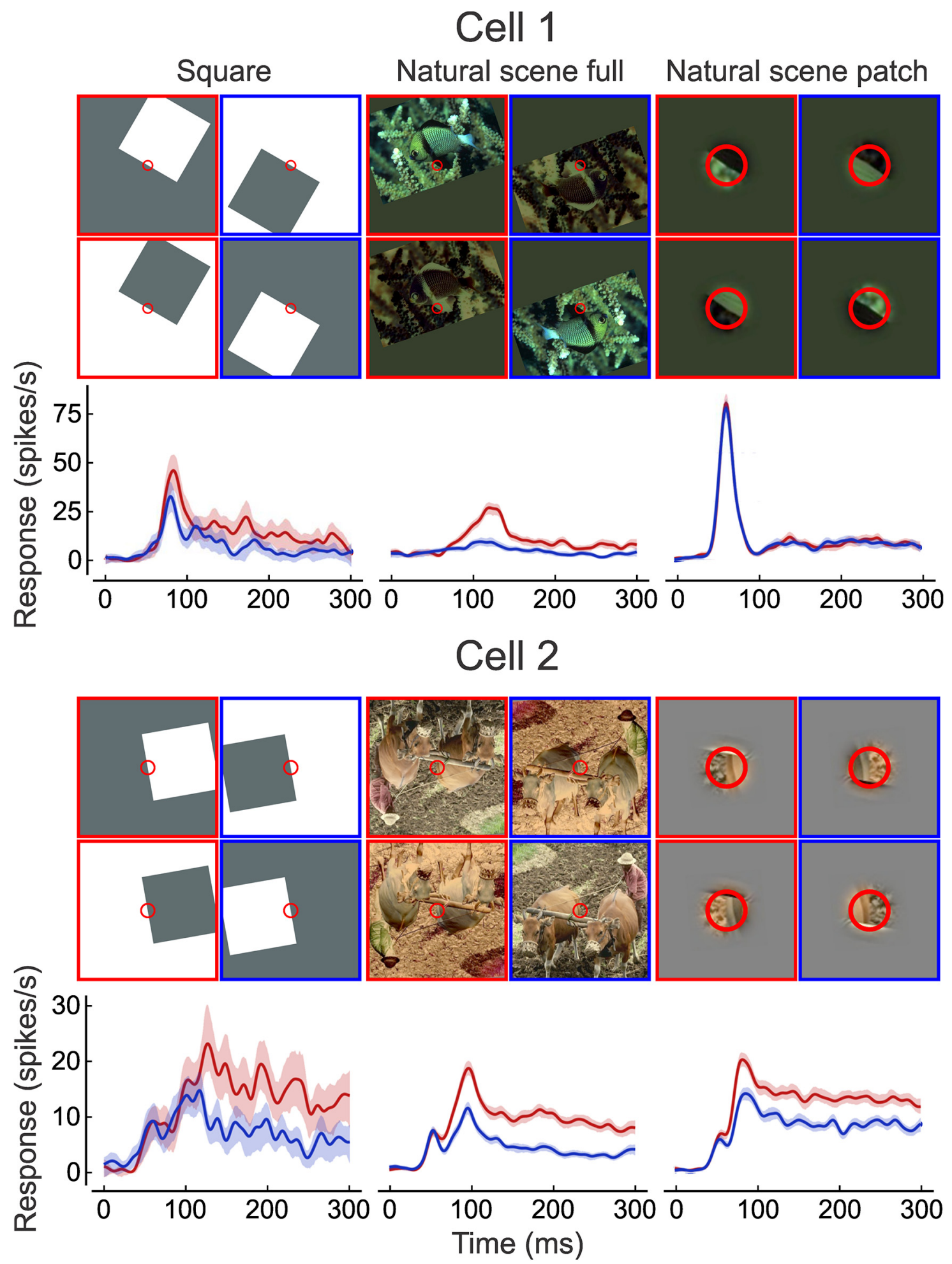

Figure 2. Responses from two example cells to square, full natural scene and patch of natural scene. Four frames are shown for each stimulus type, with the two sides of border-ownership shown side by side and the two contrast polarities at top and bottom. Red circles indicate location and approximate size of the CRF. The patch stimuli have been magnified for illustration. Red frames indicate stimuli with objects on the preferred side, and blue frames indicate stimuli on the opposite side. Only one of the presented scenes is shown in each case. Cell 1 was presented 44 scenes, and cell 2 was presented 177 scenes. The mean temporal responses after the onset of the stimulus are plotted below with the corresponding colors; shading indicates $95 \%$ confidence intervals. 
Table 1. Statistical analysis

\begin{tabular}{|c|c|c|c|}
\hline Line & Data structure & Type of test & Power \\
\hline a & Approximately normal (Anscombe transformed spike rates) & Two-sided Wilcoxon signed rank test & $p=0.87$ \\
\hline b & Binomial & One-tailed binomial & $p=0.25$ \\
\hline c & Percentage/ratio & Bootstrap & $95 \% \mathrm{Cl}: 36$ to $148 \%$ \\
\hline d & Percentage/ratio & Bootstrap & $95 \% \mathrm{Cl}: 33$ to $89 \%$ \\
\hline e & Percentage/ratio & Bootstrap & $95 \% \mathrm{Cl}:-12$ to $27 \%$ \\
\hline f & Binomial & Normal approximation of binomial test & $p=6 \times 10^{-4}$ \\
\hline$g$ & Binomial & Normal approximation of binomial test & $p=6 \times 10^{-14}$ \\
\hline
\end{tabular}

rically. The border-ownership effects were calculated using the linear models described above. Because firing rates and reliability of responses vary between cells, we plotted the effects in each cell divided by the square-root of its error variance, as obtained from the model fit to the standard square test data. Because this model contains all experimental variables and their interactions, the error variance from it reflects the variation of responses between repeated presentations of the same visual conditions. This normalization is equivalent to weighting cells by their reliability (1/error variance). The fit was forced through the origin, because the sign of the border-ownership signal for each neuron is arbitrary (two neurons responding to the same edge can have opposite directions of border-ownership preference, and which direction we assign a positive value is arbitrary; of course, the same assignment was used for all data from a given neuron). This ambiguity means that the fit must be invariant against reflecting any data points about the origin. Thus, the fitted line must pass through the origin. For the figures, we reflected the data points at the origin so as to make the effects from standard square test positive, thus plotting all results in two quadrants. The $95 \%$ confidence intervals for the slope of the line were calculated by bootstrap, resampling cells with replacement, using the $\mathrm{R}$ boot package (Davison, 1997; Canty and Ripley, 2015).

Variation of border-ownership signals across scenes

To illustrate the variation of border-ownership signals in a neuron across scenes, we sorted the scenes by the strength of the border-ownership effect, separately for full images, patches, and context. We then generated the distribution of border-ownership effects that would result if border-ownership had no influence (null hypothesis). We generated the null-hypothesis distribution from the neuron's linear model (see above) by setting the coefficients of side-of-object and its interactions to 0 , and then simulating the neuron's responses by adding random Gaussian noise with the variance equal to the residual variance of the model fit. The $95 \%$ confidence intervals of the null-hypothesis line were calculated by bootstrap, resampling scenes with replacement.

To calculate a consistency index, we compiled the neuron's distribution of border-ownership effects across scenes and deconvolved this distribution with the nullhypothesis distribution, using the DeconPdf function of the $R$ decon library (Wang and Wang, 2011). The original distribution was first approximated with a Gaussian to eliminate aliasing artifacts.

\section{Time course}

The spike rate histograms were calculated for the population of cells that were border-ownership selective in both the standard square test and the natural scenes test. The border-ownership signal is the difference between the spike rate histograms for preferred and nonpreferred side. The histograms were smoothed (Lowess, tension $0.12)$.

Latencies were determined by two-phase regression on the cumulative spike count histograms with 1-ms resolution of the population (Sugihara et al., 2011). For the overall responses, the spike counts were cumulated in the interval from 0 to $80 \mathrm{~ms}$ (Spikes pref + Spikes $\left._{\text {nonpref }}\right)$.

For the border-ownership signals, the difference between the spike counts for preferred and nonpreferred conditions were cumulated in the interval from 30 to 150 ms (Spikes $_{\text {pref }}-$ Spikes $_{\text {nonpref }}$ ).

The onset of the context effect was calculated using the cumulative differential spike counts in the full image conditions minus the cumulative differential spike counts in the patch conditions:

$$
\left(\text { Spikes }_{\text {pref }}^{\text {full }}-\text { Spikes }_{\text {nompref }}^{\text {full }}\right)-\left(\text { Spikes }_{\text {pref }}^{\text {patch }}-\text { Spikes }_{\text {nonpref }}^{\text {patch }}\right) .
$$

For the border-ownership signals, the first leg of the regression fit was forced to zero, since the activity from the preferred and nonpreferred conditions should cancel out before the onset of responses. In each case, the intersection of the regression lines defined the latency.

The standard deviations of the latencies were calculated by the bootstrap method (Efron and Tibshirani, 1993). The bootstrap was performed by resampling the stimulus presentations with replacement, within cell and condition. The number of presentations within cell and condition were kept the same as in the original data. For each resampled dataset, the latency estimates were calculated by two-phase regression as described above. Superscript letters listed with $p$-values correspond to the statistical tests shown in Table 1.

\section{Results}

We studied neurons in the visual cortices from five hemispheres of three male rhesus macaques (FR, BE, and $\mathrm{GR})$. Once spikes of a cell were isolated, its orientation and color selectivity was determined and its CRF was mapped with bars or contrast edges. Next, borderownership selectivity was measured by placing an edge of a square across the CRF center with the square located on one side or the other of the edge. Responses to both sides were recorded with either edge-contrast polarity 
(flipping the colors of square and background), and this was done for two sizes of squares $\left(3^{\circ}\right.$ and $8^{\circ}$ visual angle on a side). Flipping the side of square and edge-contrast polarity produced pairs of configurations in which the figure was on opposite sides while the edge in the CRF was identical. The border-ownership signal was defined as the response difference associated with the location of the figure. The difference averaged over the two sizes of squares is the border-ownership signal for the "standard square test." Because it compares pairs of conditions in which the stimuli are identical within the entire region defined by the two locations of the square (see Fig. 2), the standard square test measures the influence of image context outside that region.

Our goal in this study was to test neurons in early visual cortex with occluding contours in natural scenes and see whether they can signal which is the object side. How can we measure border-ownership signals elicited by natural scenes? In principle, we used the same strategy as in the standard square test. We took a sample of digitized photographs of natural scenes from a standard database (Berkeley Segmentation Dataset, Martin et al., 2001). As humans, we understand the images and can thus identify objects and their contours. We placed an occluding contour across the CRF center of a neuron, rotated the image so that the contour matched the neuron's preferred orientation, and recorded the response for this orientation and again after rotating the image $180^{\circ}$, keeping the contour in the center of the CRF. The rotation brings the object to the other side, thus reversing the direction of border-ownership in the receptive field. However, with natural contours, the situation is more complicated than with the edge of a square, because these contours are generally not straight, and the regions on both sides of the contour are not uniform. Therefore, it is impossible to compare two situations that are locally identical, as we do in the standard square test. If the contour in the CRF is curved one way in the first presentation, it will curve the other way after the rotation. Even for a straight contour, we cannot make the two tests locally identical, because the regions adjacent to the contour are not uniform. A region may have texture or contain a luminance/color gradient. Curvature, texture, and gradients are effective in determining perceived border-ownership in human vision-e.g., the concave side of a curvature is more likely to be the object side (Kanizsa, 1979), image structures that terminate at the contour are likely to be background (von der Heydt et al., 1984; Heitger et al., 1992, 1998), and if one side shows a gradient perpendicular to the contour it is likely to be the object side ("extremal edge," Palmer and Ghose, 2008). Because we cannot control these factors completely, we can only estimate border-ownership selectivity by testing each neuron with many different images. To get a better handle on the influence of local features, we expanded the factorial design by adding two dimensions of stimulus variation, patch and edgecontrast polarity.

First, in addition to presenting the entire image, we also presented a patch covering the CRF in isolation so that we could separate the effects of local and global factors.
The stimulus presented within the CRF was identical in both the entire image and patch. Outside of the CRF, the patch blended into a uniform colored surround. The surround color was set to the mean of the color values within the patch. By rotating the patch $180^{\circ}$, we determined the influence of the local border-ownership cues in isolation.

Second, to control for the effect of edge-contrast polarity, we devised a color inversion scheme analogous to that of the standard square test. This is important, because contrast polarity is sensed by the CRF, which drives the neuron, and can strongly affect the strength of responses; in V2 and the supragranular layers of V1, $\sim 50 \%$ of neurons are contrast-polarity selective (Friedman et al., 2003). To control for this factor, we created color-inverted images that essentially reversed the local edge contrast within the CRF. The test contour divides the patch into two roughly equal regions, and we calculated a transformation in $3 \mathrm{D}$ color space that flipped the mean colors of the two halves of the patch. The color values across the entire image were transformed this way. This transformation is analogous to flipping figure and ground colors in the standard square test, except that it is based on the mean colors in the vicinity of the CRF. One could of course perform the border-ownership test with only the original color images and determine the main effect of border-ownership. The contrast polarity would then be a random factor. Using color-inverted images allowed us to include contrast polarity as a covariate, which greatly improved the power of the analysis.

Each neuron was tested with many different natural scenes (10-177, mean 43) and with the two sizes of squares in the standard square test. In early experiments, we selected pieces of contours that approximately matched the preferred orientation of the neuron under study $\left( \pm 5^{\circ}\right)$ and rotated the image by a small angle to match preferred orientation exactly. Thus, we kept the images upright except for a small rotation. With this method, we had to use a large number of images, each with multiple test points. At later stages, we instead used a smaller subset of images/scene points, which enabled us to test the same set of scene points in every neuron. This implied larger rotations $\left( \pm 90^{\circ}\right)$ to match the neurons' preferred orientations. This selection of test points also excluded high local curvature and contour junctions, whereas the earlier set of images included such complex situations. Analysis of the data showed no difference between these two experimental schemes. Specifically, there was no significant difference between the firing rates in response to upright image and inverted images $(p$ $=0.87^{\mathrm{a}}$, two-sided Wilcoxon signed-rank test). We therefore pooled the results obtained with the two schemes.

We obtained complete sets of data from 140 V2 neurons of three monkeys: FR (53 neurons), BE (50 neurons), and GR (37 neurons). Of these, 88 cells $(63 \%)$ had a significant effect of border-ownership in the standard square test $(p<0.01$; see Materials and methods, Analysis of spike counts), and 65 (46\%) had a significant effect of border-ownership for objects in the full images of natural scenes. The border-ownership effect from the global context, as measured by the interaction of patch with 
Full

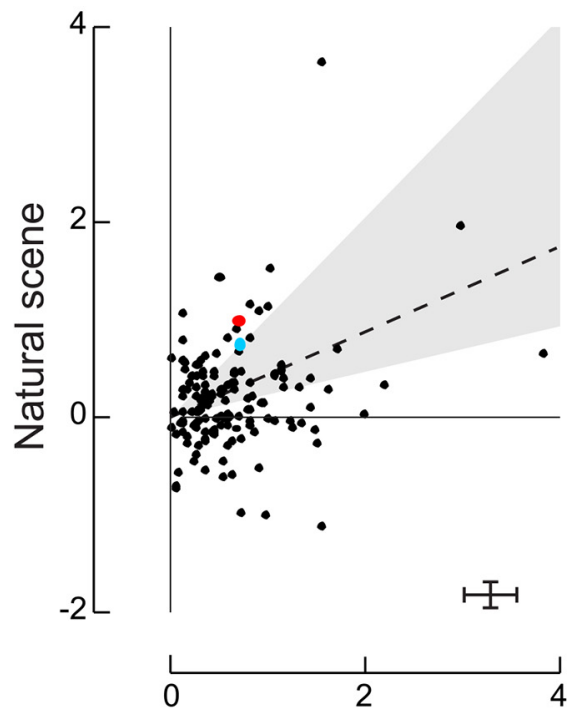

Patch

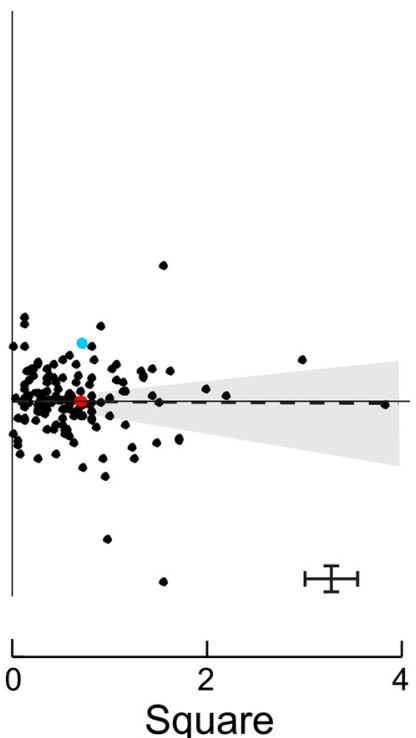

Context

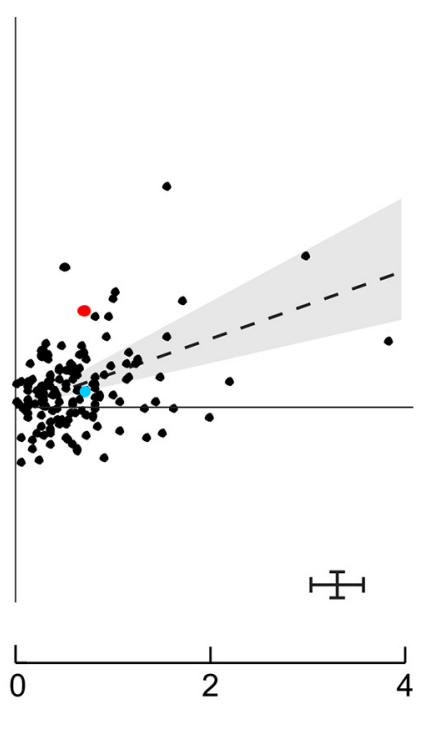

Figure 3. Comparison of border-ownership signals produced by natural scenes and squares. For each of the 140 V2 cells studied, the border-ownership effects of full image and patch, and the context influence, are plotted against the border-ownership effect of squares. The effects were measured by linear regression performed on square root-transformed spike counts (see Materials and methods). Error bars bracket the range of $6 \times$ the mean standard errors of estimates. The slopes of the lines, determined by minimizing the orthogonal squared deviations, indicate the relative strengths of border-ownership signals for the natural scene stimuli relative to squares across the population. Shaded areas and dashed lines indicate $95 \%$ confidence intervals. Colors mark the example neurons of Fig. 2 (red, Cell 1; blue, Cell 2).

border-ownership, was significant in 38 cells $(28 \%)$. Because testing natural images was sometimes skipped if border-ownership selectivity was not obvious in the standard test, these percentages may overestimate the true frequency of border-ownership selectivity.

As mentioned above, the $180^{\circ}$ image rotation did not affect the mean strength of responses across the population. Thus, the border-ownership effects in natural scenes are not the result of a response difference between upright and inverted scenes.

\section{Contours in natural scenes versus edges of squares}

How do the border-ownership signals for complex natural scenes compare with those for the much simpler standard square test displays where the object is a figure surrounded by uniform color? Because the standard square test measures the influence of context, it was of interest to compare its results with the context effect in natural scenes. In 33 cells, both the standard square test and the context of natural scenes produced significant border-ownership modulation $(p<0.01)$. Of these, only 2 cells had opposite preferences. That is, $94 \%$ of the cells were found to signal side-of-object consistently in the two tests.

The poststimulus time histograms of the responses from two example cells are illustrated in Fig. 2, along with an example scene for each (in total, Cell 1 was presented 44 scenes, and Cell 2, 177 scenes; the histograms for natural scenes are means across scenes). The conditions in which the square or natural object was on the cell's preferred side are demarked with red, and the conditions in which they were presented on the cell's nonpreferred side are demarked with blue. The preferred side is defined consistently for each cell (using the square test as the standard). Cell 1 (Fig. $2 A$ ) produced a significant $(p<$ 0.01) mean border-ownership signal with the full-image displays, which can be seen in the higher mean firing rate when the objects appeared on the preferred side (red) compared with the nonpreferred side (blue), but not with the patches. Cell 2 (Fig. 2B) produced a significant mean border-ownership signal in both full-image and patch conditions. The significant signal for patch indicates that the cell was sensitive to local figure-ground cues (e.g., curvature, edge luminance profile, texture differences). As we shall see, the behavior of Cell 1 is more representative of the population results; although there were 35 cells with significant border-ownership signals in the patch condition ( $p<0.01$ ), only 20 of these cells $(57 \%)$ were consistent between the patch condition and the standard square test (not significantly greater than chance, $p=0.25^{\mathrm{b}}$, one-tailed binomial test).

To show how the border-ownership signals for natural scenes and square relate across the population of cells, we plotted their mean signal across natural scenes versus their signal for the standard square test (Fig. 3). For each neuron, the border-ownership effects in both tests were determined by linear models and divided by the square root of the residual variance from the model of the square test (see Materials and methods). Because the model of the square test includes all variables and interactions, its residual variance reflects only random response variation. Thus, the figure represents the border-ownership signals of each neuron in multiples of the standard deviation of the random variation of its responses. Note that all signals 
of each neuron were scaled by the same factor to allow comparison between conditions.

To compare the strength of signals for contours in natural scenes with that for edges of squares, we calculated the regression lines through the origin, minimizing the orthogonal squared deviations (see Materials and methods). Minimizing absolute deviations instead of squared deviations produced similar results, as did fitting the direct effects rather than scaled effects. The positive slopes of the regression lines indicate that there was overall agreement between the results for the full images of natural scenes and the standard square test, and between the context effects of natural scenes and the standard square test. The values of the slopes also indicate that the border-ownership signals for natural scenes tended to be weaker than those for the squares. For the full natural scenes, the relative strength was $44 \%$, and for the context influence alone, it was $35 \%$. The slope for the patches was not significantly different from zero, indicating that the effects of local border-ownership cues in isolation were not consistent with the border-ownership signals evoked by the squares. Note also that the context signals were less scattered than the full-image signals. Apparently, separating the influences of local cues from the context effects reduced the variance, presumably because the effects of local cues did not correlate with the border-ownership signals for edges of squares. We checked whether the results depended on the three cells with the strongest border-ownership signals; but excluding these cells did not change the significance of the fits.

Computing the slopes for the subpopulation of cells that were border-ownership selective $(p<0.01)$ for both natural scenes and squares, the strength of the borderownership signal for natural scenes was $72 \%$ of that for squares $\left(95 \% \mathrm{Cl}: 36\right.$ to $\left.148 \%{ }^{\circ}\right)$, the influence of the context was $59 \%\left(\mathrm{Cl}: 33\right.$ to $\left.89 \%{ }^{\mathrm{d}}\right)$, and the local cues still had no effect $\left(2 \%, \mathrm{Cl}:-12\right.$ to $\left.27 \%{ }^{\mathrm{e}}\right)$.

The scatter in the plots of Fig. 3 shows that the relative strength of the border-ownership effects for natural scenes and square figures varied greatly between neurons. This is the reason for the wide confidence intervals of the fitted slopes. The scatter in these plots is not simply the result of random response variation. In fact, the confidence intervals of the effects in the individual neurons were quite small: error bars in the figure indicate $6 \times$ the mean standard errors $(\sim 99 \% \mathrm{Cl})$. Rather, the scatter indicates genuine differences between neurons in processing the two kinds of stimuli. Zhou et al. (2000) noted this variation when comparing border-ownership selectivity between different configurations of geometrical shapes and attributed it to differences between neurons in the way they evaluate the available cues. Our results, discussed in the next section, confirm this conjecture.

\section{Variation of border-ownership signals across scenes}

Above, we examined the strength of border-ownership signals looking at means across scenes. Perhaps the most intriguing question is how consistent the signals are across the different scenes. In Fig. 4 we have plotted, for the two example cells, the border-ownership effects of

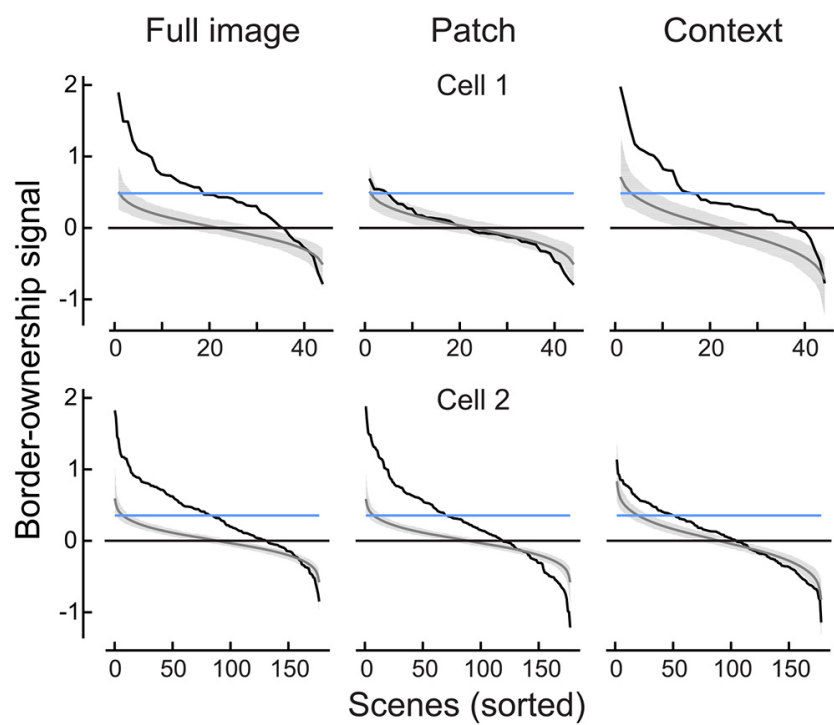

Figure 4. Variation of border-ownership signals across scenes. Data from the same cells as in Fig. 2. Scenes were sorted, in decreasing order, by strength of border-ownership signal. Black lines represent signals for full image, patch, and context. Gray bands show the $95 \%$ confidence intervals of the sorted effects obtained under the null hypothesis (no border-ownership selectivity). Horizontal blue line indicates the border-ownership signal for squares. The 10 scenes that elicited the most positive and the 10 scenes that elicited the most negative border-ownership signals are shown for each cell and condition in Figs. 4.1 and 4.2.

the individual scenes, sorted by size, for the full-image and patch conditions and for the context influence (black curves). The intersections of the curves with the abscissa are shifted to the right of the center because the majority of scenes produced positive effects, except for the patch condition in Cell 1 (Fig. 4A), which produced no consistent effects.

These plots indicate considerable variation of the effects across scenes, with strong positive effects (consistent with the standard square test) for some scenes and weak or even negative effects for others. Of course, some of this variation is caused by the random variation of responses. Determining the effects for the individual images includes substantial amounts of random variance, because the number of responses to each scene was small. We determined the effect of random variation by generating for each recorded neuron a large number of surrogate neurons with the same random variance, but no border-ownership selectivity, and sorting the predicted border-ownership effects of each surrogate neuron (see Materials and methods). The mean across the surrogate neurons represents the null hypothesis that borderownership has no influence (gray trace in Fig. 4; shading represents 95\% confidence limits). The null-hypothesis curve of course intersects the zero line near the center, as about half the values must be positive and half negative. The slope of this curve is entirely the result of sorting random variations.

Comparison with the null-hypothesis curve suggests that some of the negative values of the data are the result 
of random response variation. Particularly in the case of the patch condition in Cell 1 (Fig. 4A), the null hypothesis might explain nearly all the variation of border-ownership effects between scenes, indicating that the pictorial cues within the CRF had little, if any influence. Thus, in this neuron, the border-ownership signals in the full-image condition were driven mainly by the image context.

The random variation of responses affects the apparent consistency of the border-ownership effects across scenes. Because these variations are not correlated with the border-ownership effects, they do not systematically affect the estimate of its mean, but they tend to lower the proportion of consistent values: in the limit, when the random variance is large compared to the mean, the proportion of positive values approaches 0.5 (chance). More specifically, the measured distribution of borderownership effects is the convolution of the true distribution of border-ownership effects with the noise distribution. The latter equals the distribution of simulated border-ownership effects under the null hypothesis. Thus, in principle, we can recover the true distribution by deconvolution (see Materials and methods). Validation on simulated neurons with known border-ownership consistencies showed that the deconvolution provided a better estimate of consistency than using the raw borderownership effects, while still tending to underestimate the true consistency.

Fig. 5 shows the distribution across neurons of the proportion consistent for the full-image condition as derived from the raw data (top) and as corrected by deconvolution (bottom). The corrected results show that 13 cells were $>80 \%$ consistent across scenes, and 3 were $>90 \%$ consistent. Example Cell 1 (Fig. 2) was $83 \%$ consistent $(p$ $=6 \times 10^{-4}$, testing proportion against 0.5 , Bonferroni corrected for testing the 65 cells with significant borderownership in the full images ${ }^{f}$ ), and Cell 2 was $79 \%$ consistent $p=6 \times 10^{-14}$, testing proportion against 0.5 , Bonferroni corrected for testing the 65 cells with significant border-ownership in the full images ${ }^{9}$ ). When an occluding contour is placed in the receptive field of such a neuron, it signals the object side correctly with fairly high probability.

\section{Time course}

How can neurons at this low level in the visual cortex signal consistent figure-ground interpretations for natural scenes? Solving the figure-ground problem in complex images clearly requires some understanding of the image. Such understanding might be based on object recognition. Is it possible that the border-ownership signals for natural scenes are the result of feedback from shapeselective neurons in IT cortex? Because IT neurons start responding only $\sim 80 \mathrm{~ms}$ after stimulus onset and become shape selective at $\sim 130$ ms (Brincat and Connor, 2006, their Fig. 2, time point of half-maximal signal strength), border-ownership modulation for contours in natural scenes would then emerge with a corresponding delay, and much later than the border-ownership signals for edges of squares, which appear $\sim 70 \mathrm{~ms}$ after stimulus
A
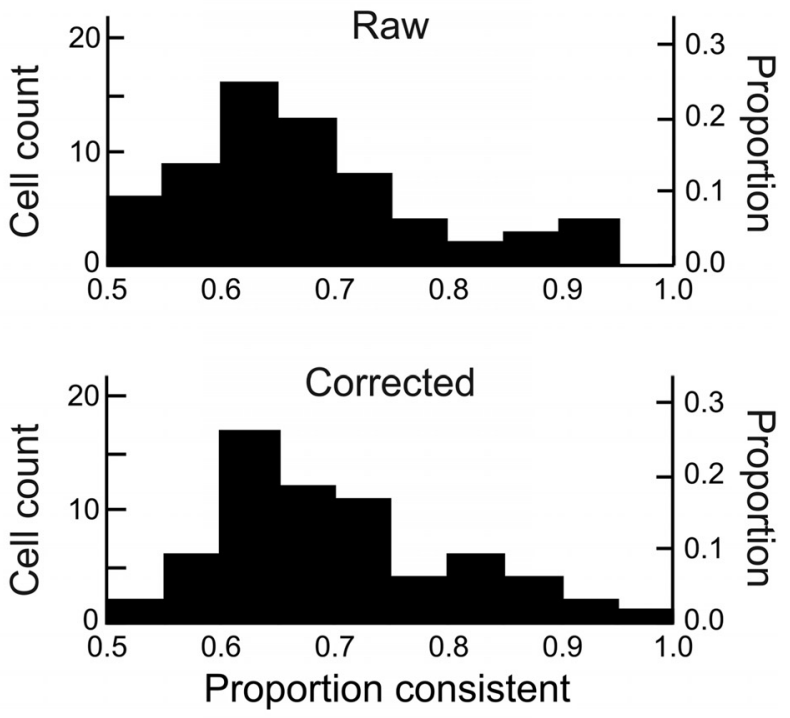

B

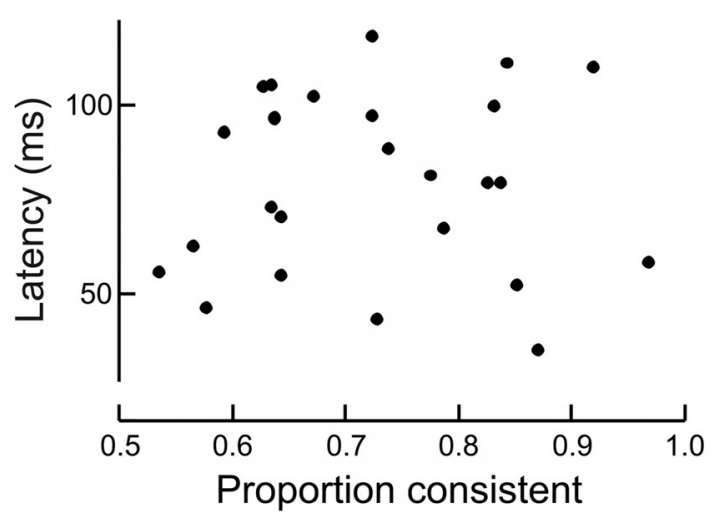

Figure 5. Consistency and latency of neurons in signaling border-ownership for natural scenes. $\boldsymbol{A}$, "Raw" shows the distribution of the proportion of scenes for which a cell gave the same sign of border-ownership signals. "Corrected" shows the distribution of the proportion after correction for random variation (see Results). Cells are selected for significant $(p<0.01)$ effect of border-ownership in full natural scenes $(n=65)$. $\boldsymbol{B}$, Estimates of the latencies of the border-ownership signals for natural scenes in the individual cells plotted as a function of their consistency. The latency of the border-ownership signals did not increase with consistency.

onset (Zhou et al., 2000; Sugihara et al., 2011). Surprisingly, this was not the case.

We computed the average poststimulus time histograms for the different stimulus conditions using the data of the 31 cells that showed significant $(p<0.01)$ effects of border-ownership for both squares and natural scenes, excluding the two cells in which the effects were opposite (because the border-ownership signal is the difference between preferred and nonpreferred side responses, cells with opposite preferences cannot be included because choosing one or the other side would bias the result toward either natural scenes or squares). A comparison of the histograms (Fig. 6A) shows that the border-ownership signals emerged virtually at the same time for natural scenes (red line) as for squares (black dashed line). 

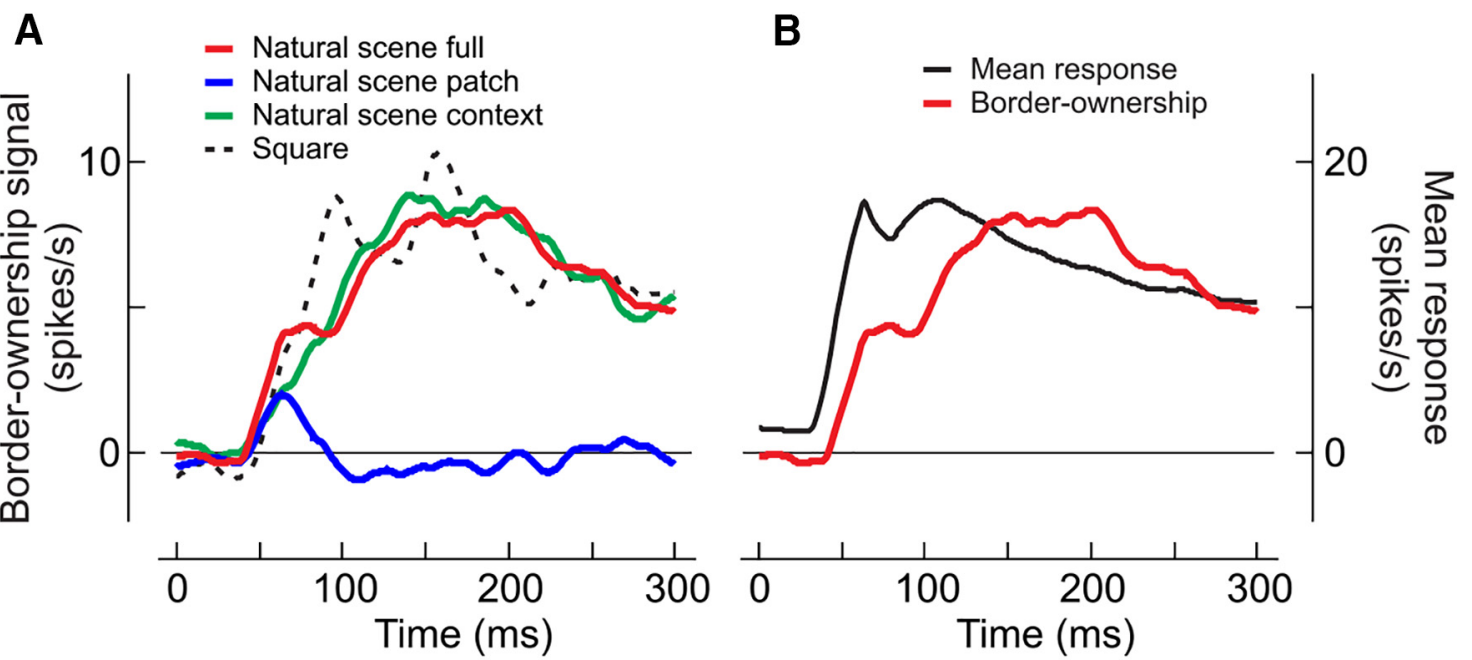

Figure 6. Time course of border-ownership signals for squares and objects in natural scenes. $\boldsymbol{A}$, The curves show smoothed poststimulus time histograms of the mean border-ownership signals, averaged over cells, for full natural scene (red), natural scene patch (blue), natural scene context (green), and square (black dashed). The border-ownership signal for natural scenes and its context component emerge at the same time as the signal for square figures, but rise slightly more slowly. The patches of natural scenes produce only a small transient signal. $\boldsymbol{B}$, Comparison of the time course of border-ownership signal (red) and mean response (black) for contours of natural scenes. Note the short interval between response onset and rise of the border-ownership signal.

We also plotted the border-ownership modulation produced by the image patches (blue) and the context effect, i.e., the difference between full-image and patch effects (green). The image patches produced only a small transient positive signal between $\sim 40$ and 90 ms which then disappeared, whereas the image context effect rose about linearly, peaking at $140 \mathrm{~ms}$. The image context effect should be compared with the border-ownership signal produced by the square (here shown for the $8^{\circ}$ size) which also depends entirely on the image context (the displays produced by flipping the square about the test edge and reversing the contrast are identical within a $16^{\circ}$ by $8^{\circ}$ region centered on the CRF). Although both emerge at the same time, one can see that the curve for natural scenes (red) rises slightly more slowly than the curve for the square (black dashed). All border-ownership signals except those for the patch lasted until the end of the observation period $(300 \mathrm{~ms})$, decaying slightly after the peak.

In Fig. 6B, we compare the border-ownership signal for natural scenes (red, replotted from $A$ ) with the mean of the responses to preferred and nonpreferred sides (black, righthand scale). Note the short delay between response onset and border-ownership signal.

The results of Fig. 6 are consistent with the scatterplots of Fig. 3 in showing that the border-ownership signals, averaged over the analysis period, did not correlate between the patch and square conditions, and that the context effects of natural scenes correlated more tightly with the square signals than the full-image signals did. Apparently, the CRF produces consistent border-ownership signals only briefly after stimulus onset, but does not have a consistent effect later.

Table 2 summarizes the latencies for responses and border-ownership signals in the different conditions as determined by two-phase regression on the cumulative spike count histograms (Fig. 7; Materials and methods). These latencies correspond approximately to the time point at half-maximal strength of the signal. The table shows a latency of $60 \mathrm{~ms}$ for the full image borderownership signal and $73 \mathrm{~ms}$ for the context influence, compared to $44 \mathrm{~ms}$ for the response onset (the response latencies were essentially the same for preferred and nonpreferred side: 44.7 and $43.8 \mathrm{~ms}$, respectively). The estimate for the response onset agrees with previous estimates for edge responses in V2 (Zhang and von der Heydt, 2010). Thus, assuming that the computation is based on V2 responses, this leaves only about $16 \mathrm{~ms}$ for computing the border-ownership signal for the full image, and $29 \mathrm{~ms}$ for evaluating the image context.

The short latency of the mean border-ownership signal for natural scenes is surprising considering the high consistency of the border-ownership effects across scenes in some neurons (Fig. 5). Could the early part of the signal be contributed by less-consistent neurons that produce strong signals, but only for a few scenes, whereas the later part of the signal comes from highly consistent neurons with longer latency (which would be compatible with feedback from IT)? Our data contradict this explanation: Cell 1 in Fig. 2 was highly consistent (83\%, 44 scenes

Table 2. Summary of the latencies of responses and borderownership signals

\begin{tabular}{lll}
\hline Stimulus & $\begin{array}{l}\text { Onset } \\
\text { response }(\mathrm{ms})\end{array}$ & $\begin{array}{l}\text { Border-ownership } \\
\text { signal }(\mathrm{ms})\end{array}$ \\
Square $\left(3^{\circ}\right)$ & $46 \pm 0.3$ & $62 \pm 3$ \\
Square $\left(8^{\circ}\right)$ & $46 \pm 0.3$ & $71 \pm 6$ \\
Natural scenes, full & $44 \pm 0.1$ & $60 \pm 2$ \\
Natural scenes, patch & $47 \pm 0.1$ & - \\
Natural scenes, context & - & $73 \pm 4$
\end{tabular}

Latencies for onset response and border-ownership signal and their standard deviations for 33 border-ownership selective cells. 


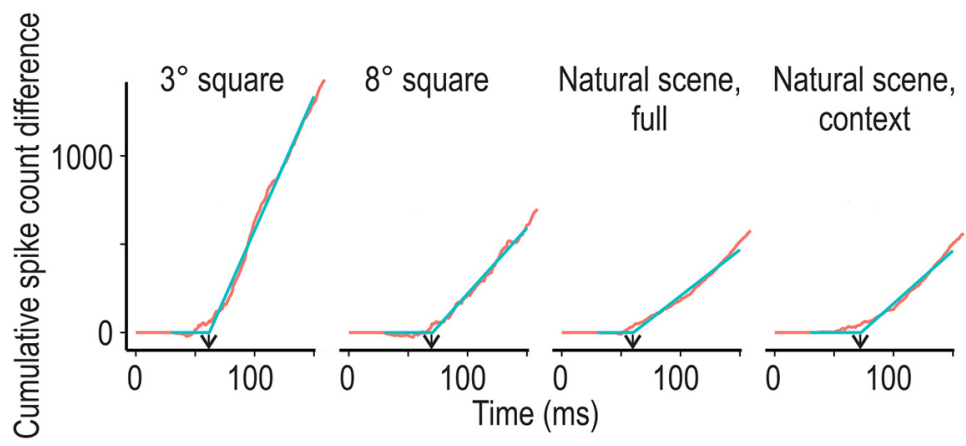

Figure 7. Determination of the latencies of the population border-ownership signals. Red curves show the cumulative spike difference counts for small and large squares, the full natural scenes, and the context component. The latencies were determined by two-phase regression fits (blue lines; see Materials and methods).

tested) and produced border-ownership modulation with short latency (85 ms). Across the population, there was no evidence of latencies being longer for highly consistent neurons (Fig. 5B). There was no correlation between Fisher-transformed consistency index and latency (Pearson $r=-0.04, p=0.85)$.
Taken together, our analysis revealed no indication of a gradual emergence of border-ownership signals for natural scenes that would correlate with the progression of shape selective responses in the ventral stream. The border-ownership signals pop up extremely fast, within $<30 \mathrm{~ms}$ after the onset of responses in visual cortex.

\section{Cell 1}

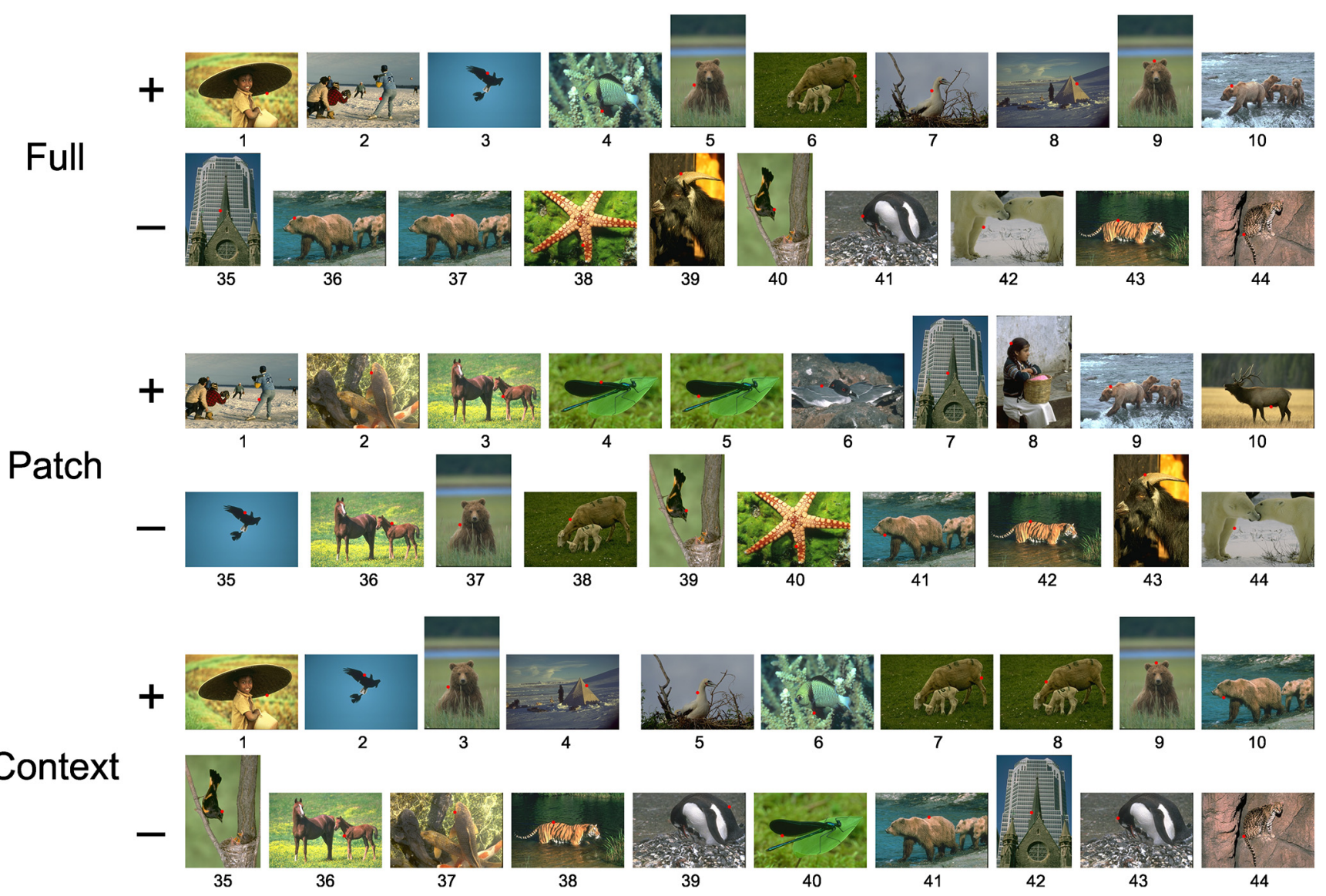

Source Figure 4. Scenes corresponding to the 10 strongest and 10 weakest border-ownership signals of Fig. 4 . The scenes were ranked according to full image, patch, and context effects for each of the two example cells. The ranks are indicated below the images: the lowest numbers correspond to the strongest signals, and the highest numbers correspond to the weakest (or negative) signals. Red dots indicate the tested receptive field positions. In some scenes, two positions were tested. Note that the scenes tend to rank similarly in full-image and context influence but differently in the patch condition. For example, in Cell 1, the eagle ranks low in both full-image and context but high in patch, and in Cell 2, the golf scene ranks low in context but high in patch. 


\section{Discussion}

This is the first neurophysiological study of borderownership selectivity of cortical neurons in natural scenes. Our main findings are that a subset of V2 cells consistently signal border-ownership in natural scenes, that the border-ownership coding for natural scenes is consistent with that for simple figure displays like a square in a uniform field, and that the border-ownership signals for natural scenes emerge at the same time as the borderownership signals for simple figures, reaching their maximal strength only slightly later. We believe our results portray a picture of the neural activity that corresponds closely to how real scenes are represented in the visual cortex under natural viewing: recording from neurons in awake monkeys during periods of fixation, we studied the responses of orientation-selective neurons (which constitute $>75 \%$ of the neurons in V2, Friedman et al., 2003) by aligning their receptive fields with occluding contours in images of natural scenes. As a consequence, the neurons generally responded strongly to at least some of the images. Thus, we are looking at the pattern of activity across the population of neurons that would be strongly activated by objects in natural scenes. These neurons presumably provide the information on which subsequent object processing centers rely.

\section{Robust mechanisms}

The border-ownership signals showed a remarkable degree of consistency, both across the population of neurons and across scenes within the individual neuron. Among the neurons that were significantly modulated by border-ownership for both natural scene contours and borders of squares (about one quarter of our sample), the mean border-ownership signals in the two conditions were consistent in $94 \%$ of the neurons. Comparing the responses of individual neurons across large samples of different natural scenes, we found that some neurons were $90 \%$ consistent across scenes. These V2 neurons perform much better than the simulated neurons in a somewhat simplistic model (Sakai et al., 2012), which reached only $67 \%$ consistency on images of the Berkeley Segmentation Dataset. We are not aware of other neural models that have been evaluated on images of natural scenes. The consistency across scenes rules out the possibility that the mean effects of border-ownership were the result of occasional conspicuous features or configurations. Finding such a high degree of consistency in neurons of a low-level visual area such as V2 is surprising.

Our results of course apply to our specific selection of scenes and test points, which is somewhat arbitrary. However, as the example set in Fig. 1 shows, our test contours included a large variety of situations: borders between regions of different contrasts, colors, and textures and borders with luminance/color gradients. Also, the shapes and sizes of the objects varied greatly. Thus, the observed consistency of border-ownership coding shows that border-ownership coding reflects mechanisms for detection of objectness that are highly sophisticated and robust.
There are many local cues that can influence our perception of border-ownership (Peterson and Salvagio, 2010). Our results from the image patches show that the CRFs did not contribute much to the border-ownership signals, except for the brief period from $\sim 50$ to $90 \mathrm{~ms}$ after stimulus onset (Fig. 6). The weakness of borderownership signals from patches is consistent with perceptual and computational studies (Fowlkes et al., 2007) showing that human performance strongly depends on the size of the image patch and would be poor or at chance level for the small patch sizes we used, especially because we excluded regions of high curvature and contour junctions. It is important to note that finding weak effects in the patch condition does not mean that local cues have little influence on the border-ownership computation. The patch generates a brief signal that is later discarded, as there is no confirmation from the context. Most likely, the border-ownership mechanisms integrate local cues all along the contours as proposed in the grouping cell model discussed below.

\section{Fast processing}

Perhaps the most surprising result is the early onset of border-ownership signals in the natural scenes test. Using two-phase regression on the cumulative differential spike count histograms, we estimated signal latencies (corresponding approximately to the time point when the border-ownership signal reaches half-maximal strength) of $60 \mathrm{~ms}$ for contours in natural scenes as a whole, and 73 $\mathrm{ms}$ for the component contributed by the image context (Table 2). These latencies are essentially the same as the latencies of the border-ownership signals for edges of squares (62 and $71 \mathrm{~ms}$ for sizes of $3^{\circ}$ and $8^{\circ}$, respectively). Because neurons in area V2 start responding at only $\sim 45$ $\mathrm{ms}$ (in all conditions), this leaves only $16-\mathrm{ms}$ processing time in the case of full images and $29 \mathrm{~ms}$ for the context influence.

\section{Searching for an explanation}

Explaining the robust and fast figure-ground signals is a challenge for the theory and modeling of visual cortical function. The spread of feed-forward connections, which account for the CRF, is too small to account for the context integration (Zhou et al., 2000; Angelucci et al., 2002). Also, lateral propagation of signals within the cortex (V1 or V2) is unlikely to explain these results, because the conduction velocity of intracortical ("horizontal") fibers is too slow to transmit the necessary context information across these large retinotopic representations, an argument that is based on an analysis of border-ownership signals for edges of squares, where the cortical distances can be determined exactly (Craft et al., 2007; Sugihara et al., 2011). By extension, given the similarity of the time course between the border-ownership responses to natural scenes and squares (Fig. 6), it seems unlikely that lateral propagation would underlie the natural scene data. This seems to rule out models based on intracortical propagation of signals (Zhaoping, 2005).

The short latency of the border-ownership signals for natural scenes also seems to rule out mechanisms tuned to specific object shapes in IT cortex as an explanation, 
because neurons there start responding only $\sim 80 \mathrm{~ms}$ after stimulus onset and become shape selective at only $\sim 130 \mathrm{~ms}$ (Brincat and Connor, 2006). Also, applying a classifier-based readout technique to the responses of many IT neurons yields valid object categorization only after $\sim 100$ ms (Hung et al., 2005). Thus, any top-down influence from specific object-recognition mechanisms would arrive much later than the observed borderownership signals.

By elimination, we conclude that border-ownership coding must involve back-projections to V1 and V2 from hypothetical higher-level mechanisms that are fast enough to generate consistent signals within a few tens of milliseconds. Large context integration occurs in a number of cortical areas that are only a few centimeters away, and, because the forward- and back-projection loops consist of white matter fibers that have high conduction velocity, this hypothesis can explain the critical observation of wide context integration with short latency.

This is the basic idea of the "grouping cell model" proposed earlier (Craft et al., 2007; Qiu et al., 2007). This model explains border-ownership coding for the relatively simple displays of geometrical figures, such as a geometrical figure surrounded by a uniform region (Zhou et al., 2000), pairs of overlapping figures (Zhou et al., 2000; Qiu et al., 2007), and the concave part of C-shaped figures (Zhou et al., 2000). In this model, neural responses from edge-selective neurons (simple or complex cells in areas V1 and V2) feed into grouping cells (G-cells) at a higher level, which, by feedback, set the gain of the same-edge neurons, thus modulating their visual responses. The feedback modulation makes these edge neurons borderownership selective (B-cells). In Craft et al. (2007), the grouping is based on very simple fixed summation templates; G-cells sum cocircular edge signals. Per design, they respond best to objects of compact shape and bias the border-ownership responses even when only a few roughly cocircular edges are present, in agreement with the neurophysiology (Zhang and von der Heydt, 2010; for psychophysics, see Elder and Zucker, 1998). The G-cells receive additional bottom-up input from end-stopped cells responding to T-junctions, which suggest occlusion and indicate the direction of occlusion (Heitger et al., 1992). Thus, G-cells accumulate evidence from various kinds of local cues distributed along the contour. However, an isolated patch containing such a cue would not be sufficient to keep a G-cell active, which explains the brief duration of the neural signal in the patch condition (Fig. 6). In strong support of this model, analysis of spike correlations between simultaneously recorded neurons Martin and von der Heydt (2015) found increased synchrony precisely between those neurons that, according to the model, receive common input from grouping cells.

A number of alternative ideas have been proposed to solve the border-ownership problem. An example is a theory by Zucker (2012) in which a field computation replaces the role of the grouping cells. In this model, edge signals are products in a reaction-diffusion equation such that, in the equilibrium state, the field carries information about distant edge arrangements (see also Cohen and
Grossberg, 1984). Zucker suggests that the local field potential is the substrate of the field computation, citing evidence that its local gradient can influence the firing rates of neurons.

It is unclear how well the different models estimate border-ownership for objects in natural scenes and how realistic they are given the extent of the cortical representation and the short latency of the border-ownership signals (Fig. 6). We are making the data from our experiments available (Williford and von der Heydt, 2016), so that border-ownership models can be compared directly to the neuronal responses.

\section{Border-ownership and saccadic eye movements}

Understanding the object structure of scenes from images is a fundamental task of vision. Our results show that this task is performed to some extent by extremely fast mechanisms that do not depend on feedback from object-recognition centers. The same mechanisms might underlie the ultrarapid object detection found by Thorpe and colleagues (Kirchner and Thorpe, 2006; Crouzet et al., 2010). When two scenes are simultaneously flashed in the left and right hemifields, human observers can reliably make saccades to the side containing an animal in as little as $120 \mathrm{~ms}$. Saccades to faces are even faster. As suggested by Martin and von der Heydt (2015), the activation of grouping circuits corresponds to the formation of "object files" or "proto-objects" postulated in perceptual theories (Kahneman et al., 1992; Rensink, 2000). The grouping circuits are thought to provide the structure for object-based attention (Craft et al., 2007; Qiu et al., 2007; Mihalas et al., 2011), which is closely related to saccade planning. Thus, the fast border-ownership signals for natural scenes demonstrated in the present study might reflect the formation of protoobjects, which also enable the system to make fast saccades to objects.

\section{References}

Angelucci A, Levitt JB, Walton EJ, Hupe JM, Bullier J, Lund JS (2002) Circuits for local and global signal integration in primary visual cortex. J Neurosci 22:8633-8646. Medline

Brincat SL, Connor CE (2006) Dynamic shape synthesis in posterior inferotemporal cortex. Neuron 49:17-24. CrossRef Medline

Canty A, Ripley BD (2015) Boot: Bootstrap R (S-Plus) Functions. R package version 1.3-15.

Cohen MA, Grossberg S (1984) Neural dynamics of brightness perception: features, boundaries, diffusion, and resonance. Percept Psychophys 36:428-456. Medline

Craft E, Schütze H, Niebur E, von der Heydt R (2007) A neural model of figure-ground organization. J Neurophysiol 97:4310-4326. CrossRef Medline

Crouzet SM, Kirchner H, Thorpe SJ (2010) Fast saccades toward faces: face detection in just $100 \mathrm{~ms}$. J Vis 10:16-17. CrossRef

Davison AC (1997) Bootstrap Methods and Their Application. Cambridge, UK: Cambridge University Press.

Efron B, Tibshirani RJ (1993) An Introduction to the Bootstrap. New York: Chapman and Hall.

Elder JH, Zucker SW (1998) Evidence for boundary-specific grouping. Vision Res 38:143-152. Medline

Fowlkes CC, Martin DR, Malik J (2007) Local figure-ground cues are valid for natural images. J Vision 7:2. CrossRef Medline

Fox J, Weisberg S (2011) An $\{R\}$ Companion to Applied Regression. Thousand Oaks, CA: Sage. 
Friedman HS, Zhou H, von der Heydt R (2003) The coding of uniform color figures in monkey visual cortex. J Physiol (Lond) 548:593613. CrossRef Medline

Heitger F, Rosenthaler L, von der Heydt R, Peterhans E, Kübler O (1992) Simulation of neuronal contour mechanisms: from simple to endstopped cells. Vision Res 32:963-981. Medline

Heitger F, von der Heydt R, Peterhans E, Rosenthaler L, Kübler O (1998) Simulation of neural contour mechanisms: representing anomalous contours. Image Vis Comput 16:409-423. CrossRef

Hung CP, Kreiman G, Poggio T, DiCarlo JJ (2005) Fast readout of object identity from macaque inferior temporal cortex. Science 310:863-866. CrossRef Medline

Kahneman D, Treisman A, Gibbs B (1992) The reviewing of object files: object-specific integration of information. Cogn Psychol 24: 175-219. Medline

Kanizsa G (1979) Organization in Vision. Essays on Gestalt Perception. New York: Praeger.

Kirchner H, Thorpe SJ (2006) Ultra-rapid object detection with saccadic eye movements: visual processing speed revisited. Vis Res 46:1762-1776. CrossRef Medline

Lamme VAF (1995) The neurophysiology of figure-ground segregation in primary visual cortex. J Neurosci 15:1605-1615.

Lee TS, Mumford D, Romero R, Lamme VAF (1998) The role of the primary visual cortex in higher level vision. Vis Res 38:2429-2454. Medline

Marcus DS, Van Essen DC (2002) Scene segmentation and attention in primate cortical areas V1 and V2. J Neurophysiol 88:2648-2658. CrossRef Medline

Martin AB, von der Heydt R (2015) Spike synchrony reveals emergence of proto-objects in visual cortex. J Neurosci 35:6860-6870. CrossRef Medline

Martin D, Fowlkes C, Tal D, Malik J (2001) A database of human segmented natural images and its application to evaluating segmentation algorithms and measuring ecological statistics. Proc 8th Int Conf Comput Vis 2:416-423.

Mihalas S, Dong Y, von der Heydt R, Niebur E (2011) Mechanisms of perceptual organization provide auto-zoom and auto-localization for attention to objects. Proc Natl Acad Sci U S A 108:7583-7588. CrossRef

Palmer SE, Ghose T (2008) Extremal edges: a powerful cue to depth perception and figure-ground organization. Psychol Sci 19:77-84. CrossRef Medline

Peterson M, Salvagio E (2010) Figure-ground perception. Scholarpedia 5:4320. CrossRef

Qiu FT, Sugihara T, von der Heydt R (2007) Figure-ground mechanisms provide structure for selective attention. Nat Neurosci 10: 1492-1499. CrossRef Medline
Qiu FT, von der Heydt R (2005) Figure and ground in the visual cortex: V2 combines stereoscopic cues with Gestalt rules. Neuron 47:155-166. CrossRef Medline

R Development Core Team (2008) R: A Language and Environment for Statistical Computing. Vienna, Austria: R Foundation for Statistical Computing.

Rensink RA (2000) Seeing, sensing, and scrutinizing. Vision Res 40:1469-1487. Medline

Russell AF, Mihalas S, von der Heydt R, Niebur E, EtienneCummings $R$ (2014) A model of proto-object based saliency. Vision Res 94:1-15. CrossRef Medline

Sakai K, Nishimura H (2006) Surrounding suppression and facilitation in the determination of border ownership. J Cogn Neurosci 18: 562-579. CrossRef Medline

Sakai K, Nishimura H, Shimizu R, Kondo K (2012) Consistent and robust determination of border ownership based on asymmetric surrounding contrast. Neural Netw 33:257-274. CrossRef Medline

Sugihara T, Qiu FT, von der Heydt R (2011) The speed of context integration in the visual cortex. J Neurophysiol 106:374-385. CrossRef Medline

von der Heydt R, Peterhans E, Baumgartner G (1984) Illusory contours and cortical neuron responses. Science 224:1260-1262. Medline

Wang XF, Wang B (2011) Deconvolution estimation in measurement error models: the R package decon. J Stat Softw 39:1-24. CrossRef

Williford JR, von der Heydt R (2013) Border-ownership coding. Scholarpedia 8:30040. Medline

Williford JR, von der Heydt R (2016) Data associated with publication "Figure-ground organization in visual cortex for natural scenes." Version 1. Johns Hopkins University Data Archive. Available at: http://dx.doi.org/10.7281/T1C8276W.

Zhang NR, von der Heydt R (2010) Analysis of the context integration mechanisms underlying figure-ground organization in the visual cortex. J Neurosci 30:6482-6496.

Zhaoping L (2005) Border ownership from intracortical interactions in visual area V2. Neuron 47:147-153.

Zhou H, Friedman HS, von der Heydt R (2000) Coding of border ownership in monkey visual cortex. J Neurosci 20:6594-6611.

Zipser K, Lamme VAF, Schiller PH (1996) Contextual modulation in primary visual cortex. J Neurosci 16:7376-7389.

Zucker SW (2012) Local field potentials and border ownership: a conjecture about computation in visual cortex. J Physiol (Paris) 106:297-315. 